\title{
A review on deep learning approaches to forecasting the changes of sea level
}

\begin{abstract}
The amalgamation of atmospheric elements indicates positive trends in sea level rise which has had a significant impact on nearly $60 \%$ of the world's population living in the low elevated coastal area. In this paper, we first discuss potential factors leading to the rise in sea level and negative impacts on future development along the coastal region. Then, methods of acquiring sea level data which revolutionize the study of variation at sea level will also be reviewed and discussed. The present paper aims to review several Deep Learning (DL) algorithms that address critical issues of forecasting, specifically a time variable known as time series by managing complex patterns and inefficiently capturing long-term multivariate data dependency. Asynchronous data handling required correct theoretical framework processes. Based on the review conducted, the deep learning architecture is capable of generating accurate prediction at sea level which can be used as decision-making tools for managing low-lying coastal areas.
\end{abstract}

